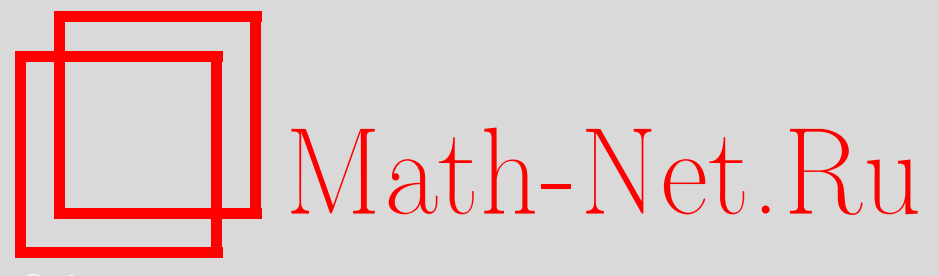

В. С. Монахов, О. А. Шпырко, О нильпотентной $\pi$-длине конечной $\pi$-разрешимой группы, Дискрет. матем., 2001, том 13, выпуск 3, 145-152

DOI: https://doi.org/10.4213/dm297

Использование Общероссийского математического портала Math-Net.Ru подразумевает, что вы прочитали и согласны с пользовательским соглашением http://www. mathnet.ru/rus/agreement

Параметры загрузки:

IP: 54.210 .77 .194

26 апреля 2023 г., 12:50:13

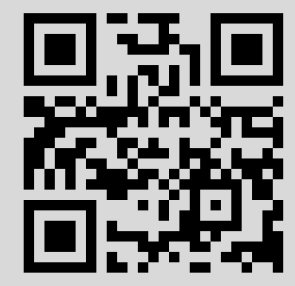




\title{
О нильпотентной $\pi$-длине конечной $\pi$-разрешимой группы
}

\author{
(C) 2001 г. $\quad$ В. С. Монахов, О. А. Шпырко
}

Получены новые оценки нильпотентной $\pi$-длины конечной $\pi$-разрешимой группы, у которой коммутант $\pi$-холловой подгруппы нильпотентен. Установлена связь между нильпотентной $\pi$-длиной конечной $\pi$-разрешимой группы и производной длиной ее холловой $\pi$-подгруппы нечетного порядка.

Для непустой формации $\mathfrak{F}$ в [1] определена $\mathfrak{F}$-длина конечной группы, которая при конкретных значениях $\mathfrak{F}$ приводит, в частности, к понятиям $\pi$-длины и нильпотентной $\pi$-длины $\pi$-разрешимой-группы. Напомним эти определения. Рассматриваются только конечные группы. Пусть $\pi$ - некоторое множество простых чисел. Через $\pi^{\prime}$ обозначают множество всех простых чисел, не содержащихся в $\pi$, а через $\pi(G)-$ множество простых чисел, делящих порядок группы $G$. Возрастающим $\left(\pi^{\prime}, \pi\right)$-рядом группы $G$ называют ряд

$$
1=P_{0} \leqslant N_{0} \leqslant P_{1} \leqslant N_{1} \leqslant \ldots,
$$

где $N_{i} / P_{i}=O_{\pi^{\prime}}\left(G / P_{i}\right), P_{i+1} / N_{i}=O_{\pi}\left(G / N_{i}\right), i=0,1, \ldots$ Здесь $O_{\pi^{\prime}}(X)$ и $O_{\pi}(X)-$ наибольшие нормальные $\pi^{\prime}$ - и $\pi$-подгруппы группы $X$ соответственно. Если группа $G \pi$-разрешима, то $N_{k}=G$ для некоторого натурального $k$. Наименьшее натуральное $k$ с этим свойством называют $\pi$-длиной $\pi$-разрешимой группы $G$ и обозначают через $l_{\pi}(G)$.

Нильпотентная $\pi$-длина $\pi$-разрешимой группы $G$ определяется следующим образом. Пусть

$$
P_{0}^{n}=1, \quad N_{i}^{n} / P_{i}^{n}=O_{\pi^{\prime}}\left(G / P_{i}^{n}\right), \quad P_{i+1}^{n} / N_{i}^{n}=F\left(G / N_{i}^{n}\right), \quad i=0,1, \ldots
$$

Здесь $F(X)$ - подгруппа Фиттинга группы $X$. Наименьшее $k$, для которого в ряде

$$
1=P_{0}^{n} \leqslant N_{0}^{n} \leqslant P_{1}^{n} \leqslant N_{1}^{n} \leqslant \ldots
$$

выполняется равенство $N_{k}^{n}=G$, называется нильпотентной $\pi$-длиной группы $G$ и обозначается через $l_{\pi}^{n}(G)$. Поскольку $P_{i+1}^{n} / N_{i}^{n}-$ нильпотентная $\pi$-группа, а $N_{i}^{n} / P_{i}^{n}$ - $\pi^{\prime}$-группа, то $l_{\pi}(G) \leqslant l_{\pi}^{n}(G)$, а если $\pi=\{p\}$, то $l_{\pi}^{n}(G)=l_{\pi}(G)=l_{p}(G)$. Ясно также, что равенство $l_{\pi}(G)=l_{\pi}^{n}(G)$ сохраняется для $\pi$-разрешимой группы с нильпотентной $\pi$-холловой подгруппой.

С понятием $\pi$-длины связана следующая проблема Л. А. Шеметкова (см. [2], вопрос 11.119): верно ли, что для любого непустого множества $\pi$ простых чисел 
$\pi$-длина $\pi$-разрешимой группы ограничена сверху ступенью разрешимости ее $\pi$-холловой подгруппы? Эта проблема рассматривалась в работах $[3,4,5]$, где получен положительный ответ, в случае, когда $2 \notin \pi$, (см. [5], теорема 1). Заметим, что этот результат легко переносится на нильпотентную $\pi$-длину (см. теорему 1 настоящей работы). Кроме того, в настоящей работе получены новые оценки $\pi$-длины и нильпотентной $\pi$-длины $\pi$-разрешимой группы в зависимости от строения ее силовских $p$-подгрупп для $p \in \pi$.

Для индуктивных рассуждений нам потребуются следующие леммы. Как и в [4], под $l_{\pi}^{*}(G)$ понимается либо всюду $l_{\pi}(G)$, либо всюду $l_{\pi}^{n}(G)$.

Лемма 1 ([3], леммы 1,2; [4], лемма 1). Пустъ $\pi$ - некоторое множество простых чисел и $G-\pi$-разрешимая группа. Тогда

(1) если $H-$ подгруппа группъ $G$, mo $l_{\pi}^{*}(H) \leqslant l_{\pi}^{*}(G)$,

(2) если $N$ - нормальная подгруппа группь $G$, mо $l_{\pi}^{*}(G / N) \leqslant l_{\pi}^{*}(G)$, если $N-$ нормалъная $\pi^{\prime}$-подеруппа группы $G$, mo $l_{\pi}^{*}(G)=l_{\pi}^{*}(G / N)$,

(3) в любом ряде нормалъных подгрупп группы $G$ с $\pi^{\prime}$-факторами и $\pi$-факторами (нилъпотентными $\pi$-факторами) число неединичных $\pi$-бакторов не менъше, чем $l_{\pi}(G)$ (не менъше, чем $l_{\pi}^{n}(G)$ ),

(4) если $H_{i} \triangleleft G, i=1, \ldots, n u G=H_{1} H_{2} \ldots H_{n}, \operatorname{mol}_{\pi}^{*}(G)=\max _{i=1, \ldots, n} l_{\pi}^{*}\left(H_{i}\right)$.

Лемма 2. Пусть $\pi$ - некоторое множество простых чисел $u G-\pi$-разрешимая группа. Eсли $l_{\pi}^{*}(G / N)<l_{\pi}^{*}(G)$ для всех неединичных нормалънъх подгрупп $N$ әрупnъ $G, m o$

(1) $O_{\pi^{\prime}}(G)=\Phi(G)=1$,

(2) в группе $G$ единственная минимальная нормальная подгруппа $K$,

(3) $K$ - элементарная абелева $p$-группа, $p \in \pi, u K=O_{p^{\prime}, p}(G)=P_{1}^{n}$,

(4) $C_{G}(K)=K$ u подгруппа $K$ дополняема в группе $G$.

Доказательство. Отметим, что для $\pi=\{p\}$ лемма 2 совпадает с леммой VI.6.9 в [6]. Для $l_{\pi}(G)$ аналогичное утверждение содержится в лемме 4 из [3]. Поэтому остается доказать утверждения для $l_{\pi}^{n}(G)$.

Из второго утверждения леммы 1 следует, что $O_{\pi^{\prime}}(G)=1$. Так как подгруппа Фраттини $\Phi=\Phi(G)$ нильпотентна, то $\Phi-\pi$-подгруппа и $\Phi \leqslant P_{1}^{n}=F(G)$ в обозначениях ряда (1) для группы $G$. Если $\Phi-$ собственная подгруппа в $P_{1}^{n}$, то из определения нильпотентной $\pi$-длины следует, что $l_{\pi}^{n}(G)=l_{\pi}^{n}(G / \Phi)$ и $\Phi=1$ по условию леммы. Пусть $\Phi=P_{1}^{n}$. Тогда по теореме Шура-Цассенхауза $N_{1}=H \Phi$, где $H$ - неединичная $\pi^{\prime}$-подгруппа. По лемме Фраттини $G=N_{G}(H) \Phi$, а по свойствам подгруппы Фраттини $G=N_{G}(H)$. Теперь $1 \neq H \leqslant O_{\pi^{\prime}}(G)=1$. Поэтому допущение $\Phi=P_{1}^{n}$ неверно и первое утверждение доказано.

Допустим, что в группе $G$ две минимальные нормальные подгруппы $N_{1}$ и $N_{2}$. Тогда $N_{1} \cap N_{2}=1$ и по лемме Ремака группа $G$ изоморфна подгруппе прямого произведения $G / N_{1} \times G / N_{2}$. Теперь

$$
l_{\pi}^{n}(G) \leqslant \max _{i=1,2} l_{\pi}^{n}\left(G / N_{i}\right)
$$


согласно первому и четвертому утверждениям леммы. Получили противоречие с условием доказываемой леммы. Поэтому допущение неверно и второе утверждение леммы справедливо.

Так как $O_{\pi^{\prime}}(G)=1$ и группа $G \pi$-разрешима, то $K$ - элементарная абелева $p$-подгруппа, $p \in \pi$. Из второго утверждения следует, что $O_{p^{\prime}}(G)=1$, поэтому $K=O_{p^{\prime}, p}(G)=P_{1}^{n}$ по свойству подгруппы Фиттинга (см. теорему III.4.5 в [6]).

Докажем четвертое утверждение. Так как $\Phi(G)=1$, существует максимальная подгруппа $M$ в $G$ такая, что $G=M K$. Из абелевости $K$ следует, что $M \cap K=1$, то есть $M-$ дополнение к подгруппе $K$ в группе $G$. Теперь $C_{G}(K) \cap M \triangleleft G$ и в силу второго утверждения $C_{G}(K) \cap M=1$ и $K=C_{G}(K)$. Лемма доказана.

Для группы $G$ можно построить нормальный ряд

$$
1=F_{0}(G) \leqslant F_{1}(G) \leqslant \ldots \leqslant F_{k}(G) \leqslant \ldots,
$$

где $F_{k}(G) / F_{k-1}(G)=F\left(G / F_{k-1}(G)\right)$ - подгруппа Фиттинга группы $G / F_{k-1}(G)$, $k=1,2, \ldots$ Если группа $G$ разрешима, то $F_{t}(G)=G$ для некоторого $t$. Наименьшее $t$, для которого $F_{t}(G)=G$, называется нильпотентной длиной разрешимой группы $G$ и обозначается через $n(G)$. Ясно, что любой нормальный ряд группы $G$ с нильпотентными факторами имеет длину не менее, чем $n(G)$.

Лемма 3. Если $G-\pi$-разрешимая группа $u H-$ eе $\pi$-холлова подгруппа, то $n(H) \leqslant l_{\pi}^{n}(G)$.

Доказательство. Рассмотрим $\left(\pi^{\prime}, \pi\right)$-ряд группы $G$

$$
1=P_{0}^{n} \leqslant N_{0}^{n} \leqslant P_{1}^{n} \leqslant N_{1}^{n} \leqslant \ldots \leqslant N_{t}^{n}=G .
$$

Здесь $t$ - наименьшее число, для которого $N_{t}^{n}=G$, то есть $t=l_{\pi}^{n}(G)$. Факторы $P_{i+1} / P_{i}$ этого ряда обладают нормальной $\pi^{\prime}$-холловой подгруппой и нильпотентной $\pi$-холловой подгруппой. Пересекая члены этого ряда с подгруппой $H$, получим нормальный ряд подгруппы $H$

$$
\begin{aligned}
1=P_{0}^{n} \cap H & =N_{0}^{n} \cap H \leqslant P_{1}^{n} \cap H=N_{1}^{n} \cap H \leqslant \ldots \leqslant P_{i}^{n} \cap H \\
& =N_{i}^{n} \cap H \leqslant \ldots \leqslant P_{t}^{n} \cap H=N_{t}^{n} \cap H=G \cap H=H .
\end{aligned}
$$

Группа $P_{i}^{n}\left(P_{i+1}^{n} \cap H\right) / P_{i}^{n}$ является $\pi$-подгруппой группы $P_{i+1}^{n} / P_{i}^{n}$, поэтому она нильпотентна. Кроме того,

$$
P_{i}^{n}\left(P_{i+1}^{n} \cap H\right) / P_{i}^{n} \simeq\left(P_{i+1}^{n} \cap H\right) /\left(P_{i}^{n} \cap H\right),
$$

значит, построенный для $H$ ряд нормальный и его факторы нильпотентны. Так как любой нормальный ряд группы $H$ с нильпотентными факторами имеет длину не менее, чем $n(H)$, то $n(H) \leqslant t$. Лемма доказана.

Следующая лемма показывает, что для $\pi$-разрешимых групп с абелевыми силовскими $p$-подгруппами для всех $p \in \pi$ указанное в лемме 3 неравенство является точным.

Лемма 4. Нилъпотентная $\pi$-длина $\pi$-разрешимой группы с абелевыми силовскими $p$-подеруппами для всех $p \in \pi$ совпадает с нильпотентной длиной своей $\pi$-холловой nodzpynnъ. 
Доказательство. Пусть $G-\pi$-разрешимая группа с абелевыми силовскими $p$-подгруппами для всех $p \in \pi$. Зафиксируем $\pi$-холлову подгруппу $H$ группы $G$. Пусть $n(H)$ - нильпотентная длина подгруппы $H$. Из леммы 3 следует, что $n(H) \leqslant l_{\pi}^{n}(G)$. Докажем индукцией по порядку группы $G$, что $l_{\pi}^{n}(G) \leqslant n(H)$. В силу лемм 1,2 можно считать, что $O_{\pi^{\prime}}(G)=\Phi(G)=1$ и $F=F(G)$ - единственная минимальная нормальная подгруппа группы $G$, причем $F=C_{G}(F)$ и $F$ дополняема в группе $G$. Ясно, что $F$ является $p$-группой для $p \in \pi$ и $F=O_{p}(G)$. Из того, что $F=C_{G}(F)$, следует равенство $O_{p^{\prime}}(G)=1$.

Так как $F \leqslant H$ и силовская $p$-подгруппа абелева, то $F$ является силовской $p$ подгруппой группы $G$. Поэтому $F-$ подгруппа Фиттинга группы $H$. Теперь по индукции

$$
l_{\pi}^{n}(G)=l_{\pi}^{n}(G / F)+1 \leqslant n(H / F)+1=n(H)-1+1=n(H) .
$$

Лемма доказана.

Через $X^{\prime}$ обозначается коммутант группы $X$, а $X^{(i)}=\left(X^{(i-1)}\right)^{\prime}-$ eе $i$-й коммутант. Наименьшее натуральное число $t$, для которого $X^{(t)}=1$, называется ступенью разрешимости группы $X$ и обозначается через $d(X)$.

Лемма 5 ([5], лемма 9). Пустъ $H-\pi$-холлова подгруппа разрешимой группъ $G u$ nусть $O_{\pi^{\prime}}(G)=1$. Ecлu $2 \notin \pi$, mo $H^{(t-1)} \leqslant F(G)$, где $t=d(H)$.

Лемма 6. Пусть $G-\pi$-разрешимая группа, $q \in \pi^{\prime} u \omega=\pi \cup\{q\}$. Тогда в группе $G$ существуют $\omega$-холловы подгруппы и любые две $\omega$-холловы подгруппъ сопряжены между собой.

Доказательство. Утверждение леммы является частным случаем теоремы D5 из [7].

Доказательство следующей теоремы во многом повторяет доказательство теоремы 1 из [5].

Теорема 1. Если $H-\pi$-холлова подгруппа $\pi$-разрешимой группы $G$ u $2 \notin \pi$, то $l_{\pi}^{n}(G) \leqslant d(H)$.

Доказательство. Проведем доказательство индукцией по порядку группы. В силу лемм 1, 2 можно считать, что $O_{\pi^{\prime}}(G)=\Phi(G)=1$ и $F=F(G)$ - единственная минимальная нормальная подгруппа группы $G$, причем $F=C_{G}(F)$ и $F$ дополняема в группе $G$. Ясно, что $F$ является $p$-группой для $p \in \pi$ и $F=O_{p}(G)$. Из того, что $F=C_{G}(F)$, следует равенство $O_{p^{\prime}}(G)=1$. Зафиксируем $\pi$-холлову подгруппу $H$ группы $G$.

Пусть $\pi(G) \backslash \pi=\left\{r_{1}, \ldots, r_{n}\right\}$ и $\omega_{i}=\pi \cup\left\{r_{i}\right\}$. По лемме 6 в группе $G$ существует $\omega_{i-}$ холлова подгруппа и все $\omega_{i}$-холловы подгруппы сопряжены между собой. Поэтому можно выбрать $\omega_{i}$-холлову подгруппу $H_{i}$ группы $G$ так, что $H \leqslant H_{i}$. Ясно, что $H_{i}=H P_{i}$, где $P_{i}$ - некоторая силовская $r_{i}$-подгруппа группы $G$ и $H_{i}$ - разрешимая подгруппа. Так как $F \leqslant H_{i}$ и $F=C_{G}(F)$, то $O_{\pi^{\prime}}\left(H_{i}\right)=1$ и $F\left(H_{i}\right)$ - p-группа, поэтому $F\left(H_{i}\right) \leqslant F(H)$.

Пусть $t=d(H)$ и $K=H^{(t-1)}$. Тогда $K \leqslant F\left(H_{i}\right)$ по лемме 5. Теперь

$$
K^{y} \leqslant F\left(H_{i}\right)^{y}=F\left(H_{i}\right) \leqslant F(H)
$$


для всех $y \in P_{i}$. Отсюда следует, что $K^{x} \leqslant F(H)$ для всех $x \in<H, P_{1}, \ldots, P_{n}>=G$. Поэтому $K^{G}=<K^{x} \mid x \in G>$ является $p$-группой и $K \leqslant F$. По индукции

$$
l_{\pi}^{n}(G)=l_{\pi}^{n}(G / F)+1 \leqslant d(H / F)+1=d(H)-1+1=d(H) .
$$

Теорема доказана.

Лемма 7. Пусть $G-\pi$-разрешимая группа и $H-$ eе $\pi$-холлова подгруппа. Если $N \triangleleft G, m o(H N / N)^{\prime} \simeq H^{\prime} /\left(H^{\prime} \cap N\right)$.

Доказателъство. Так как $H N / N \simeq H /(N \cap H)$, то их коммутанты изоморфны. По свойствам коммутантов

$$
(H /(H \cap N))^{\prime}=H^{\prime}(H \cap N) /(H \cap N) \simeq H^{\prime} /\left(H^{\prime} \cap N\right)
$$

(см. лемму I.8.4 в [6]), поэтому $(H N / N)^{\prime} \simeq H^{\prime} /\left(H^{\prime} \cap N\right)$.

Теорема 2. Если $G$ - $\pi$-разрешимая группа, у которой коммутант $\pi$-холловой подаруппы нилъпотентен, то

$$
l_{\pi}^{n}(G) \leqslant 1+\max _{p \in \pi} l_{p}(G) .
$$

Доказателъство. Воспользуемся индукцией по порядку группы $G$. В силу лемм 1,7 условия доказываемой леммы переносятся на факторгруппы, поэтому по лемме 2 подгруппы $O_{\pi^{\prime}}(G)=\Phi(G)=1$ и в группе $G$ существует единственная минимальная нормальная подгруппа $F=P_{1}^{n}=O_{q^{\prime}, q}(G)$ для некоторого $q \in \pi$. Ясно, что $F \leqslant G_{\pi}$.

Пусть $K$ - коммутант $\pi$-холловой подгруппы $G_{\pi}$. По условию теоремы подгруппа $K$ нильпотентна. Поскольку $q^{\prime}$-холлова подгруппа $K_{q^{\prime}}$ из $K$ нормальна в $G_{\pi}$, то $K_{q^{\prime}} \leqslant C_{G}(F)=F$ и $K_{q^{\prime}}=1$. Таким образом, $K$ является $q$-группой и все силовские $p$-подгруппы в группе $G$ абелевы для $p \in \pi \backslash\{q\}$. Поэтому $l_{p}(G)=1$ для всех $p$ из $\pi \backslash\{q\}$ (см. теорему VI.6.6 в [6]) и $\max _{p \in \pi} l_{p}(G)=l_{q}(G)$.

Если $F$ - силовская подгруппа группы $G$, то в факторгруппе $G / F \pi$-холлова подгруппа $G_{\pi} / F$ абелева и $l_{\pi}^{n}(G / F)=1$ по лемме 4. Поэтому

$$
l_{\pi}^{n}(G) \leqslant 2 \leqslant 1+\max _{p \in \pi} l_{p}(G)
$$

В этом случае теорема доказана.

Пусть $F$ не является силовской подгруппой группы $G$. Тогда $l_{q}(G)=l_{q}(G / F)+1$. Теперь, применяя индукцию, получаем, что

$$
\begin{aligned}
l_{\pi}^{n}(G) & =l_{\pi}^{n}(G / F)+1 \leqslant 1+\max _{p \in \pi} l_{p}(G / F)+1 \\
& =1+l_{q}(G / F)+1=1+l_{q}(G)=1+\max _{p \in \pi} l_{p}(G) .
\end{aligned}
$$

Таким образом, неравенство $l_{\pi}^{n}(G) \leqslant 1+\max _{p \in \pi} l_{p}(G)$ доказано.

Следствие 1. Если $G-\pi$-разрешимая группа со сверхразрешимой $\pi$-холловой подгруппой, $\operatorname{mo}_{\pi}^{n}(G) \leqslant 1+\max _{p \in \pi} l_{p}(G)$.

Доказателъство. Достаточно вспомнить, что у сверхразрешимой группы коммутант нильпотентен (см. теорему VI.9.1 в.[6]), а затем применить теорему. 
Следствие 2. Если в $\pi$-разрешимой группе $G$ силовские p-подгруппы циклические для всех $p \in \pi, \operatorname{mo} l_{\pi}^{n}(G) \leqslant 2$.

Доказательство. Так как $\pi$-холлова подгруппа группы $G$ сверхразрешима по теореме VI.2.11 из [6], а по теореме VI.6.6 из [6] $p$-длина $l_{p}(G)=1$ для всех $p \in \pi$, то $l_{\pi}^{n}(G) \leqslant 2$.

Лемма 8. Если $G$ - р-разрешимая группа с метациклической силовской $p$-подгруппой $u p>2$, mo $l_{p}(G) \leqslant 1$.

Доказательство. Поскольку $l_{p}^{n}(G)=l_{p}(G)$, то применимы леммы 1 и 2 . По индукции подгруппа Фраттини единична и в группе $G$ единственная минимальная нормальная подгруппа. Кроме того, в группе $G$ нет неединичных нормальных $p^{\prime}-$ подгрупп. Поэтому $P=O_{p}(G)$ - элементарная абелева $p$-подгруппа, совпадающая со своим централизатором и имеющая в $G$ дополнение. Поскольку $P$ метациклическая, порядок $P$ равен $p$ или $p^{2}$. Если $|P|=p$, то $G / P$ - циклическая группа порядка, делящего $p-1$, и $P$ силовская в $G$. Если $|P|=p^{2}$, то $G / P$ изоморфна подгруппе $G L(2, p)$ и силовская $p$-подгруппа $G_{p}$ имеет порядок $p^{3}$. Если $G_{p}$ абелева, то $G_{p}=P$ по теореме VI.6.6 из [6]. Если $G_{p}$ неабелева, то по теоремам 5.4.5 и 5.4.4 из [8] подгруппа $P$ недополняема в $G_{p}$, получаем противоречие. Лемма доказана.

Лемма 9. Группа нечетного порядка с метациклическими силовскими р-подгруппами для всех р имеет нилъпотентный коммутант.

Дохазательство. Воспользуемся индукцией по порядку группы. Пусть $G$ - группа наименьшего нечетного порядка с метациклическими силовскими $p$-подгруппами для всех $p$, у которой коммутант ненильпотентен. По следствию 4.2 .1 из [1] подгруппа Фраттини группы $G$ единична. Ясно, что в группе $G$ минимальная нормальная подгруппа единственна. Поэтому подгруппа Фиттинга $F=F(G)-$ минимальная нормальная подгруппа, совпадающая со своим централизатором и дополняемая в группе $G$. По лемме 8 подгруппа $F$ силовская порядка $p$ или $p^{2}$. Если $|F|=p$, то $G / F$ циклическая порядка делящего $p-1$. Если $|F|=p^{2}$, то $G / F$ - неприводимая подгруппа нечетного порядка группы $G L(2, p)$, и опять $G / F$ циклическая. Следовательно, всегда $G^{\prime} \leqslant F$, поэтому всегда коммутант $G^{\prime}$ нильпотентен. Лемма доказана.

Лемма 10. Если $G$ - сверхразрешимая группа, то $l_{\pi}(G) \leqslant 1 u l_{\pi}^{n}(G) \leqslant 2 \partial \Omega \Omega$ любого множества $\pi$ простьх чисел.

Доказательство. Пусть $H=G^{\prime}$ - коммутант группы $G$. Так как $H$ - нильпотентная подгруппа, то $H=H_{\pi} \times H_{\pi^{\prime}} \triangleleft G$. Из того, что $H / H_{\pi^{\prime}} \triangleleft G / H_{\pi^{\prime}}$ и $G / H$ абелева, следует, что группа $G$ обладает следующим нормальным $\left(\pi^{\prime}, \pi\right)$-рядом:

$$
1 \triangleleft H_{\pi^{\prime}} \triangleleft H \triangleleft G_{\pi} H_{\pi^{\prime}} \triangleleft G \text {. }
$$

Так как факторы $H_{\pi^{\prime}}$ и $G / G_{\pi} H_{\pi^{\prime}}$ этого ряда являются $\pi^{\prime}$-группами, а факторы $H / H_{\pi^{\prime}}$ и $G_{\pi} H_{\pi^{\prime}} / H$ - нильпотентными $\pi$-группами, то $l_{\pi}^{n}(G) \leqslant 2$ и $l_{\pi}(G) \leqslant 1$. Лемма доказана.

Сверхразрешимым корадикалом группы называется наименьшая нормальная подгруппа, факторгруппа по которой сверхразрешима. 
Лемма 11. Если $H$ - неприводимая разрешимая подгруппа группы $G L(2, p)$, то сверхразрешимый корадикал подгруппы $H$ является расширением циклической 2группы порядка, делящего $p-1$, с помощъю подгруппы из элементарной абелевой группы порядка 4.

Доказательство. Заключим $H$ в максимальную разрешимую подгруппу $M$ группы $G L(2, p)$ и докажем утверждение леммы для $M$. Ясно, что тогда утверждение леммы будет справедливо и для $H$. Очевидно, $M$ - неприводимая подгруппа.

Если $M$ импримитивна, то $M$ изоморфна сплетению циклической группы $A$ порядка $p-1$ и группы $B$ порядка 2 (см. [9], теорему 18.5). Диагональ $S$ базы $D=A \times A$ этого сплетения будет нормальной подгруппой в $M$, изоморфной $A$, поэтому $M$ обладает нормальным рядом $1 \triangleleft S \triangleleft D \triangleleft M$, где $S$ и $D / S$ циклические порядка $p-1$ и $M / D$ порядка 2. Поэтому $M$ сверхразрешима.

Если $M$ примитивна и ее максимальная абелева нормальная подгруппа $F$ изоморфна мультипликативной группе поля $G F\left(p^{2}\right)$, то по теореме 21.1 из [9] факторгруппа $M / F$ имеет порядок 2 и $M$ опять сверхразрешима.

Пусть $M$ примитивна и ее максимальная абелева нормальная подгруппа $F=$ $G F(p)^{\#} E_{2}$. Подгруппа $F$ состоит из скалярных матриц ([9], с. 234, формула (13)), поэтому $F$ содержится в центре $M$. В $M$ существует нормальная подгруппа $A$ такая, что $A$ содержит $F$ и $A / F$ изоморфна элементарной абелевой группе порядка 4 , а $M / A$ изоморфна симметрической группе степени 3 (см. [9], с.234, формулы (13) и (14)). Так как $A$ нильпотентна, силовская 2-подгруппа $T$ из $A$ нормальна в $M$. Теперь факторгруппа $M / T$ обладает нормальным рядом $1 \triangleleft A / T \triangleleft M / T$, который уплотняется до главного ряда с факторами простых порядков. Значит, $M / T$ сверхразрешима и сверхразрешимый корадикал содержится в $T$. Но $A=T F$, поэтому $A / F \simeq T / T \cap F$ и $T$ является расширением циклической группы $T \cap F$ порядка, делящего $p-1$, с помощью элементарной абелевой группы $A / F$ порядка 4. По [9] (см. гл.V, §21) других ситуаций для $M$ нет. Лемма доказана.

Напомним, что бициклической называют конечную группу, являющуюся произведением двух циклических подгрупп. Метациклическая группа - это группа, которая содержит циклическую нормальную подгруппу с циклической факторгруппой. Метациклическая группа всегда является бициклической. Обратное утверждение верно для примарных групп нечетного порядка и не всегда выполняется для бициклических 2-групп ([6], теорема III.11.5).

Лемма 12 ([12], лемма). Любая нормальная подгруппа бичиклической 2-группы, имеющая дополнение, порождается одним или двумя элементами.

Теорема 3. Пусть $G-\pi$-разрешимая группа с бициклическими силовскими рподгруппами для всех $p \in \pi$. Тогда $l_{\pi}^{n}(G) \leqslant 4$, a $l_{\pi}(G) \leqslant 2$, u если $2 \notin \pi$, mо $_{\pi}^{n}(G) \leqslant 2$.

Доказателъство. Доказательство теоремы проводим индукцией по порядку группы $G$. В силу леммы 1 условия теоремы переносятся на факторгруппы, поэтому по лемме 2 подгруппы $O_{\pi^{\prime}}(G)=\Phi(G)=1$ и в группе $G$ существует единственная минимальная нормальная элементарная абелева $p$-подгруппа $F=P_{1}^{n}=O_{p^{\prime}, p}(G)$ для некоторого $p \in \pi$, совпадающая со своим централизатором и дополняемая в группе $G$. Ясно, что $F \leqslant G_{p}$.

Если $2 \notin \pi$, то лемма 9 позволяет применить теорему 2 , поэтому с учетом леммы 8 получаем, что $l_{\pi}^{n}(G) \leqslant 2$. 
Пусть $2 \in \pi$. Тогда $G$ - разрешимая группа: Предположим, что $p>2$. По лемме 8 подгруппа $F$ силовская в группе $G$. Так как $F$ метациклическая ([6], теорема III.11.5), то $|F|=p$ или $p^{2}$.

Если $|F|=p$, то факторгруппа $G / F$ изоморфна подгруппе циклической группы Aut $F$, порядок которой равен $p-1$. Теперь группа $G$ сверхразрешима, $l_{\pi}(G) \leqslant 1$ и $l_{\pi}^{n}(G) \leqslant 2$ по лемме 11 .

Пусть $|F|=p^{2}$. Тогда факторгруппа $G / F$ изоморфна подгруппе полной линейной группы $G L(2, p)$. Если $G / F$ - сверхразрешимая группа, то по лемме $10 l_{\pi}(G / F) \leqslant 1$ и $l_{\pi}^{n}(G / F) \leqslant 2$, отсюда $l_{\pi}(G) \leqslant 2$ и $l_{\pi}^{n}(G) \leqslant 3$. Если $G / F-$ несверхразрешимая группа, то по лемме 11 сверхразрешимый корадикал $H / F$ факторгруппы $G / F$ является 2 -группой. Учитывая, что $2 \in \pi$, заключаем с учетом леммы 10 , что $l_{\pi}(G) \leqslant 2$ и $l_{\pi}^{n}(G) \leqslant 4$.

Пусть теперь $F-2$-группа. По лемме 12 подгруппа $F$ имеет порядок 2 или 4. Если $|F|=2$, то $|G|=2$. Пусть $|F|=4$. Тогда факторгруппа $G / F$ изоморфна подгруппе группы $G L(2,2) \simeq S_{3}$ и $G / F$ сверхразрешима. Ввиду леммы 10 заключаем, что $l_{\pi}(G) \leqslant 2$, а $l_{\pi}^{n}(G) \leqslant 3$. Теорема доказана полностью.

С учетом леммы 3 при $\pi=\pi(G)$ из теоремы 3 получаем два следствия.

Следствие 3. Разрешимая группа с бичиклическими силовскими р-подгруппами для всех р имеет нилъпотентную длину не въше 4.

Следствие 4. Группа нечетного порядка с метациклическими силовскими р-подгруппами для всех р имеет нильпотентную длину не выше 2.

\section{Список литературы}

1. Шеметков Л. А., Формации конечнъх групп. Наука, Москва, 1978.

2. Нерешенные вопросъ теории групп. Коуровская тетрадъ. ИМ СО РАН, Новосибирск, 1999.

3. Черников Н. С., Петравчук А. П., Характеризация периодических локально разрешимых групп с разрешимыми и с конечноэкспонентными силовскими $\pi$-подгруппами. Украинский матем. ж. (1987) 39, №6, 761-767.

4. Черников Н. С., Петравчук А. П., О $\pi$-длине конечных $\pi$-разрешимых групп. Бесконечные группы и примыкающие алгебрачческие структуры. Институт математики, Киев, 1993, 393-405.

5. Kazarin L. S., Soluble products of groups. In: Infinite groups 94, Newell, Berlin, 1995, pp. 111-123.

6. Huppert B., Endliche Gruppen. I. Springer, Berlin, 1967.

7. Hall P., Theorems like Sylow's. Proc. London Math. Soc. (1956) 6, 286-304.

8. Gorenstein D., Finite groups. Harper and Row, New York, 1968.

9. Супруненко Д. А., Группъ матриц. Наука, Москва, 1972.

10. Maier R., Über die 2-nilpotentz faktorisierbarer endlicher Gruppen. Arch. Math. (1976) 27, №5, 480-483. 